\title{
Genome-wide identification of TCF7L2/TCF4 target miRNAs reveals a role for miR-21 in Wnt-driven epithelial cancer
}

\author{
FENGMING LAN ${ }^{1-3^{*}}$, XIAO YUE ${ }^{1-3,5^{*}}$, LEI HAN $^{1-3}$, ZHENDONG SHI $^{1-3}$, \\ YANG YANG $^{1-3}$, PEIYU PU ${ }^{1-3}$, ZHI YAO $^{4}$ and CHUNSHENG KANG $^{1-3}$ \\ ${ }^{1}$ Laboratory of Neuro-Oncology, Tianjin Neurological Institute; ${ }^{2}$ Department of Neurosurgery, Tianjin Medical University \\ General Hospital; ${ }^{3}$ Key Laboratory of Neurotrauma, Variation and Regeneration, Ministry of Education and Tianjin \\ Municipal Government; ${ }^{4}$ Department of Immunology, Basic Medical College at Tianjin Medical University, Tianjin 300052; \\ ${ }^{5}$ Department of Neurosurgery, Shandong Cancer Hospital, Shandong Academy of Medical Sciences, Jinan 250117, P.R. China
}

Received July 26, 2011; Accepted September 12, 2011

DOI: 10.3892/ijo.2011.1215

\begin{abstract}
Transcription factor 7 like 2 (TCF7L2, also known as TCF4) is a Wnt signaling pathway transcription factor involved in regulation of numerous Wnt targeted genes. Recently, thousands of high-confidence TCF4 binding sites were reported in LS174T colon carcinoma cells, however, potential TCF4 target miRNAs remain largely unknown. Here, we utilized a bioinformatics approach to discover 26 miRNA transcription start sites (TSSs) within close proximity to TCF4 chromatin occupancy sites, and validated these sites as bona fide TCF4 targets in LS174T colon carcinoma cells, MCF-7 breast cancer cells and U87 glioma cells by ChIP-PCR. We then selected miR-21 to demonstrate for the first time direct TCF4 transcriptional activation of a miRNA via binding to the promoter region. Tissue array analysis supported this finding, revealing a positive correlation between activation of the $\beta$-catenin pathway and in situ expression of miR-21. Finally, based upon the well known but poorly understood preventive effect of aspirin on colorectal cancer incidence and mortality, we report downregulation of miR-21 upon administration of aspirin. In sum, our findings identify direct transcriptional regulation of miR-21 by TCF4 and suggest a role for miR-21 in cancer cell proliferation and invasion upon activation of $\beta$-catenin/TCF4 signaling.
\end{abstract}

\section{Introduction}

Transcription factor 7 like 2 (TCF7L2, also called and hereafter referred to as TCF4) is a $76-\mathrm{kDa}$ protein encoded by a single

Correspondence to: Dr Chunsheng Kang, Laboratory of NeuroOncology, Tianjin Neurological Institute, 152 Anshan Road, Heping, Tianjin 300052, P.R. China

E-mail:kang97061@yahoo.com

*Contributed equally

Key words: $\beta$-catenin/TCF4 signaling, transcription initiation site, miRNA, miR-21 exon. The N-terminal 53 amino acids of TCF4 bind with high affinity to $\beta$-catenin, forming the $\mathrm{TCF} / \beta$-catenin transcription complex that regulates transcriptional responses to $\mathrm{Wnt}$ signaling (1). Aberrant activation of Wnt signaling results in a transcriptional profile in human cancer cells similar to that found in cells physiologically driven by $\mathrm{TCF} / \beta$-catenin, such as intestinal stem/progenitor cells (2). Amongst the TCF/ $\beta$-catenin regulated genes found overexpressed in cancers many are involved in survival pathways, including EGFR, STAT3, C-myc, and MMP2/9 $(3,4)$. TCF4 chromatin occupancy appears to function both as a developmental transcription factor necessary for progenitor cell development and as the primary transforming factor in human tumors such as colorectal cancer.

miRNA have been implicated in the pathogenesis of several human diseases, such as neurodegenerative disorders, diabetes and more recently in viral and metabolic diseases $(5,6)$. In recent years, miRNAs have been identified in the progression of various cancers and proposed as novel targets for anticancer therapies $(7,8)$. Although miRNAs are key regulators of gene expression in both normal human physiology and disease, and thousands of microRNAs have been identified in organisms from viruses to primates through cloning and sequencing, or computational prediction based on strong conservation of miRNA sequence motifs, the transcriptional regulation of miRNA are poorly understood $(9,10)$. In the present work, we perform a TCF4 binding localization analysis to identify miRNAs that have TCF4 bound at their proximal promoters. Our results detected 26 miRNAs that are high-confidence direct TCF4 targets. Further, we validated these findings by demonstrating that TCF4 influences miR-21 expression by direct binding to the promoter region. These experiments provide new insight into how TCF4 affects miRNA expression, and how these changes in miRNA expression may influence cancer progression.

\section{Materials and methods}

TCF4 peak analyses. High-confidence TCF4-binding sites $(\mathrm{n}=6,868)$, previously identified by Hatzis et al (1), were chosen for analysis. Binding site peaks located within $10 \mathrm{~kb}$ of transcription initiation sites were selected as likely to impact transcription regulation. 
Table I. The sequences of the ChIP-PCR primers.

\begin{tabular}{|c|c|c|}
\hline miRNA name & Upper primer & Lower primer \\
\hline hsa-miR-933 & 5'-TAA GGA GAA GAA GGC GGG TCT 3' & 5'-AGC CGA CAG CCA ATC ACA-3' \\
\hline hsa-miR-153-1 & 5'-GGT TCC GCA GGT GAT CCT C-3' & 5'-TCT CCA CGG GAC ACA TGA TTC-3' \\
\hline hsa-miR-561 & 5'-GGC AAA CTC CAA GTA AGT GA-3' & 5'-GTA TTT TAA AAG CAG GCA CAA-3' \\
\hline hsa-miR-566 & 5'-GCT GTG TCT AGG GCC TCG GAT-3' & 5'-TGG CGC ACA CAT GTC CTA CC-3' \\
\hline hsa-miR-297 & 5'-AAA GGA GCC ACA GAG ATT ATA-3' & 5'-ACT AGG CCA ATG GTT ATG TT-3' \\
\hline hsa-miR-584 & 5'-GAC CGA GGC CTG CTC AAT G-3' & 5'-GGC TGA TAG GCA TGA AGC GAT-3' \\
\hline hsa-miR-548a-2 & 5'-TGA CTG CTC TTC CTC CTA ACT-3' & 5'-AGA AGA TTA TCT CAC GGC TCT-3' \\
\hline hsa-miR-2113 & 5'-TGC TGC TGA CAC AGA ATC CA-3' & 5'-GGT GAA GCC AGC TAT GCA AAC-3' \\
\hline hsa-miR-219-1 & 5'-ACA GCT GTA AAC CCC GTA GTG-3' & 5'-TCA GCT CCT GGT TCC CTA TC-3' \\
\hline hsa-miR-1975 & 5'-GCA CGC GAG TGA CGG GAT T-3' & 5'-GCA TTG TGG GTA GCG CCT AGC-3' \\
\hline hsa-miR-29b-1 & 5'-TTT CAG CTT CCC AGG ATA CCC-3' & 5'-CGT GAC TTC CCG CCA GA-3' \\
\hline hsa-miR-23b & 5'-CCG CCC AGT GGA ATT CCT-3' & 5'-GGG CCC CTG TAA ATG ACA TCA-3' \\
\hline hsa-miR-7-1 & 5'-CCC ACT GCC CGA GAC AAA G-3' & 5'-TGA GCT GGA GCT ATG TGG CAC-3' \\
\hline hsa-miR-2110 & 5'-CAG GAC GCT CTT GTT GGC TAT-3' & 5'-CGA ACG CGC TGT TAT GGA-3' \\
\hline hsa-miR-936 & 5'-GCA GGA GGT CAG TGG TTG AGA-3' & 5'-GCC TGG AGT GGA GGA TAA GGA-3' \\
\hline hsa-miR-146b & 5'-GCC TTG GAG GAC ACA TGA CGA-3' & 5'-CCC AGG GTA CCC GTC CGA T-3' \\
\hline hsa-miR-2110 & 5'-TGA GTC TAA CGC GGA TGT TGT-3' & 5'-ACA CCT GCT GTC CGA AAT G-3' \\
\hline hsa-miR-615 & 5'-TGA ACC CCG GGA TGT ACA GT-3' & 5'-AGT TTA CCG TGG CTC ATG TGC-3' \\
\hline hsa-miR-1252 & 5'-CTA AAC TGT GGA GTG GCA TAG-3' & 5'-CTT GGC CTT CGT GGT T-3' \\
\hline hsa-miR-1252 & 5'-CCT CTT TCT TCT TGG CAT CA-3' & 5'-AGG GAC AGC AAG TAT CAC CTC-3' \\
\hline hsa-miR-1293 & 5'-GCA TGG AGG CAT CTG TAA-3' & 5'-GTA CCA GGG ATC TAA CTT GGA-3' \\
\hline hsa-miR-548h-1 & 5'-CAG GCA GGT GGA TCA AGC-3' & 5'-TCC GGA GTC AGT CAA ACC TTA-3' \\
\hline hsa-miR-10a & 5'-AAG CTG TGA CAG AGT AAG GGA-3' & 5'-GTG TTG CAG TTA GAG GGT TGT-3' \\
\hline hsa-miR-21 & 5'-CAA AGA TCA CTA TCC CAA TCA TC-3' & 5'-GCG GTC TTT CTC AAT CTA AGT C-3' \\
\hline hsa-miR-301a & 5'-TTC TTG GGA CGC TTA GGG ACC-3' & 5'-CAT CCG GGA AAC TCG TCA GG-3' \\
\hline hsa-miR-935 & 5'-GAT CAC AGC AGG ATG ACA TCA-3' & 5'-CTG CCC TGA CTG GTC TAA AAC-3' \\
\hline hsa-miR-103-2 & 5'-CCT TCA AGA ACC TGT TAG GTG-3' & 5'-GAG GGC AAA GGC TAC AAT-3' \\
\hline hsa-miR-424 & 5'-AGA GAT TAC AAA GGG GCG TCT-3' & 5'-TGA ACA GAG GAA GAG GCG TAT-3' \\
\hline
\end{tabular}

ChIP-PCR. LS174T colon carcinoma, U87 glioma and MCF-7 breast cancer cells purchased from the Institute of Biochemistry and Cell Biology, Chinese Academy of Science were employed in ChIP-PCR. Chromatin immunoprecipitation was conducted using the chromatin immunoprecipitation assay kit (Millipore 17-371) and manipulated according to the manufacturer's instructions. LS174T, U87 and MSF-7 cells were cross-linked with $1 \%$ formaldehyde for $20 \mathrm{~min}$ at room temperature. The cells were successively washed with phosphate-buffered saline at $4^{\circ} \mathrm{C}$ for $10 \mathrm{~min}$ each. The ChIP incubation buffer were used to resuspended cells which were then sonicated at $5 \mathrm{~W}^{*}$ for 12 pulses of $10 \mathrm{sec}$ each using a VirSonic sonicator. The sonicated chromatin was centrifuged for $15 \mathrm{~min}$ and incubated for $12 \mathrm{~h}$ at $4^{\circ} \mathrm{C}$ with either non-immune IgG (negative control), Tcf-4 (Millipore, 05-511), and Anti-RNA Polymerase II (positive control) at 1 $\mu \mathrm{g}$ of antibody per $10^{6}$ cells with protein $\mathrm{G}$ beads. The beads were successively washed 2 times with low salt wash buffer 1 , one time with high salt wash buffer 2 , and one time with $\mathrm{LiCi}$ wash buffer 3 and two times with Tris-EDTA buffer 4 for 5 min each at $4^{\circ} \mathrm{C}$. The immune chromatin complexes were eluted by incubation of the beads with elution buffer at room temperature for $20 \mathrm{~min}$, de-cross-linked by incubation at $65^{\circ} \mathrm{C}$ for $5 \mathrm{~h}$ in the presence of $200 \mathrm{mM} \mathrm{NaCl}$, extracted with phenol-chloroform, and precipitated. Purified DNA was subjected to PCR, and all sequences of the PCR primers used can be found in Table I. Two primers were chosen for each gene, in order to perform a perfect PCR, which needs to enhance specificity, primers were designed using the oligo 6 software.

Immunohistochemistry analysis. A colon cancer tissue microarray containing 120 patient samples was purchased from Chao Ying, Shanxi, China. $\beta$-catenin and TCF4 were stained with mouse anti- $\beta$-catenin and anti-TCF4 antibodies (Abcam 1:200 dilution), respectively, and detected following incubation with tetramethyl rhodamine isothiocyanate-conjugated goat secondary anti-mouse immunoglobulin G (IgG; Sigma 1:100 dilution). Sections were then incubated with ABC-peroxidase and DAB (diaminobenzidine), counterstained with hematoxylin, and visualized using light microscope (Olympus).

miR-21 detection by in situ hybridization. The in situ hybridization detection of miR-21 in colon cancer tissue microarray 
samples was performed with in situ hybridization kit (Boster, Wuhan, China). LNA/DNA oligos contained locked nucleic acids at eight consecutive centrally located bases (indicated by the underline) and had the following sequences: LNA-miR-21 5'-TCAACATCAGTCTGATAAGCTA-3'. The in situ hybridization detection of miR-21 in tissue microarray was conducted according to the protocol of the manufacturer.

TOP-FOP reporter assay. To detect the $\beta$-catenin/Tcf- 4 activity, we used a pair of luciferase reporter constructs, TOP-FLASH and FOP-FLASH (Upstate). TOP-FLASH (with three repeats of the Tcf-binding site) or FOP-FLASH (with three repeats of a mutated Tcf-binding site) plasmids were transfected into LS174T colon cancer cells which was treated with aspirin. Luciferase activity was measured by the Dual-luciferase reporter assay system, with the Renilla luciferase activity as an internal control, $48 \mathrm{~h}$ after transfection.

Luciferase assays. Cells were cultured in 96-well plates in the presence of medium (DMEM supplemented with $10 \%$ fetal calf serum), or medium supplemented with $10 \mathrm{mM}$ aspirin. Cells were transfected with pGL3-miR21-promoter or pGL3-basic vector reporter. Following 48-h incubation, luciferase activity was measured using a dual-luciferase reporter system (Promega).

Quantitative PCR ( $q P C R)$. Four hours following incubation with $10 \mathrm{mM}$ aspirin, total RNA was isolated from LS174T cells by the TRizol method. Reverse transcription was performed using a reverse transcription kit (Qiagen), and qPCR was used to detect the changes in miR-21 expression. U6 expression was utilized as a control. The primers were synthesized by Gene Pharma, Shanghai, China.

Western blot analysis. Parental and transfected cells were washed three times with pre-chilled phosphate-buffered saline (PBS). Separated proteins were transferred to PVDF membranes (Millipore, Bedford, MA, USA), and incubated with primary antibodies against PTEN, RECK and EGFR (Santa Cruz), followed by detection with an HRP-conjugated secondary antibody (Zymed). The membrane was stripped and re-probed with a primary antibody against GAPDH (Santa Cruz).

Statistical analysis. Data were expressed as mean \pm SE. Statistical analyses were determined by ANOVA, $\chi^{2}$ test, or Student's t-test using SPSS11.0 (Windows). Statistical significance was determined as ${ }^{*} \mathrm{P}<0.05$ or ${ }^{* *} \mathrm{P}<0.01$.

\section{Results}

Identification of putative TCF4 peaks within $10 \mathrm{~kb}$ of miRNA genes. To globally identify all direct TCF4-targeted genes, ChIP analysis was performed on LS174T colorectal carcinoma cells. Based on the median mean signal and the median variance of the dedicated array, 6,868 TCF4 peaks were observed (1). The hg17 Genome Browser was previously used to identify coordinate positions of the 6,868 peaks (1). Here, we utilized the hg19 Genome Browser to annotate gene positions. TCF4 binding regions within $10 \mathrm{~kb}$ of transcription initiation sites were considered likely to impact transcription regulation, and were selected for further analysis. Our findings identified 1793 peaks distributed within $10 \mathrm{~kb}$ of coding genes, and an additional 45 peaks in within $10 \mathrm{~kb}$ of miRNA coding regions (Fig. 1). Next, we analyzed the selected TCF4 binding peaks for expression of the A-C/G-A/T-T-C-A-A-A-G TCF4 binding motif. After extraction of $\sim-500$ to $+500 \mathrm{bp}$ from each peak position, we used patmatch (ftp://ftp.arabidopsis.org/ home/tair/Software/Patmatch/) to predict putative A-C/G-A/TT-C-A-A-A-G motifs, with allowances for two mismatches. Our results indicate that every identified TCF4 peak contained this motif on the basis of mismatch requirements. These findings support the hypothesis that the identified TCF4 peaks represent specific DNA sequence bound with TCF4.

Stepwise differential expression rank analysis identified a significant correlation between TCF4 occupancy of target-gene regulatory regions and gene transcription (Fig. 2). To determine whether TCF4 can regulate miRNA expression, we analyzed the association between peak location and human annotated miRNA-encoding genes.

TCF4-binding regions are distributed in clusters surrounding putative target genes. Hatzis et al demonstrated that TCF4 associated with as many as 11 peaks within $100 \mathrm{~kb}$ of the TSS of AXIN2, a well-known target gene of the Wnt pathway. Analysis of the 1793 peaks associated with protein-coding genes identified a considerable portion of genes associated with two or more TCF4 peaks. In fact, 132 genes displayed 2 TCF4 binding sites, 20 genes displayed 3 TCF4 peaks, and 5 genes displayed 4 TCF4 peaks. Based upon these data, we identified 1606 protein-coding genes within $10 \mathrm{~kb}$ distance of TCF4 peaks. Interestingly, many TCF4 binding sites are distributed in close proximity to miRNA transcription initiation sites. Analysis identified 45 putative peaks that potentially influenced the expression of 38 distinct miRNA (5 miRNA were clustered by 2 TCF4 peaks, and hsa-miR-2110 was clustered by 3 peaks). These data suggest that TCF4 may signal via transcriptional regulation of both coding and non-coding (such as miRNAs) genes.

TCF4 binds to the putative sites in the promoter element of 26 distinct miRNA. Through TCF4 tiling array analysis, we obtained TSSs of 38 miRNA found within $10 \mathrm{~kb}$ of TCF4 binding sites. Our calculations, however, included both upstream and downstream ranges of TCF4 occupancy. As TCF4 can potentially regulate miRNA expression by binding with their promoters upstream of an open reading frame (OPR), we selected 26 miRNAs whose transcription initiation sites are located downstream of TCF4 bound regions ( 28 peaks, including has-miR-2110 and hsa-miR-1252 that each contained 2 upstream TCF4 binding sites within $10 \mathrm{~kb}$ ). Each miRNA was validated by conventional ChIP. To determine whether the TCF4 binding sites associated with these 26 miRNAs are enriched in TCF4-bound promoter regions, we performed ChIP-PCR in U87, LS174T and MCF-7 cells. Primers were designed specifically around each peak, and enrichment was performed using Oligo 6 software (described in Table I). Despite inconsistency in miRNA expression among U87, LS174T and MCF-7 cells, our data strongly suggest that TCF4 interacts with the promoter regions of miRNA. Further, immunodetection identified fivefold increase in TCF4 expression compared to negative controls (Fig. 3). These data are highly provocative, as many of the miRNA identified, including miR-21, miR-10a, miR-23b and miR-566, are found to be overexpressed in many human cancers (11-21). 
A

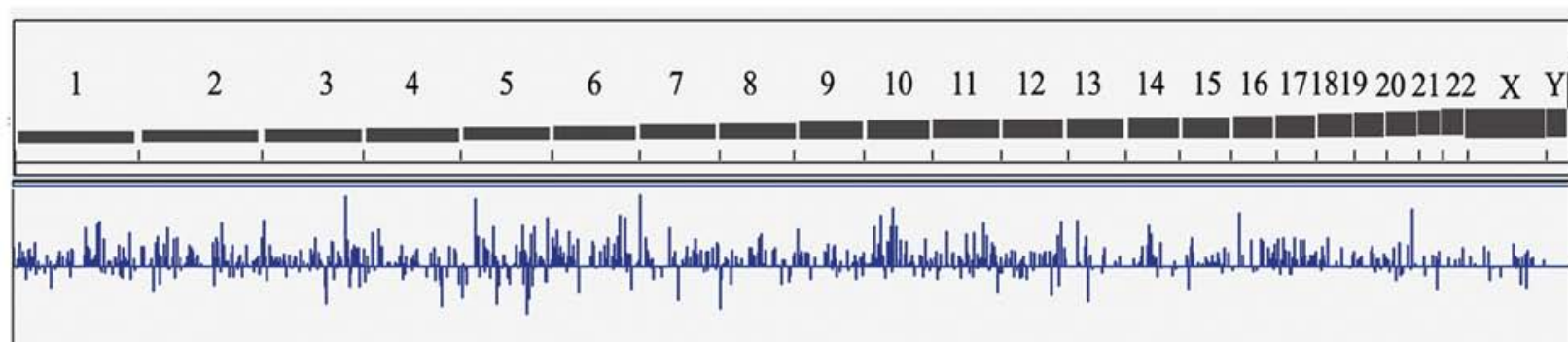

B

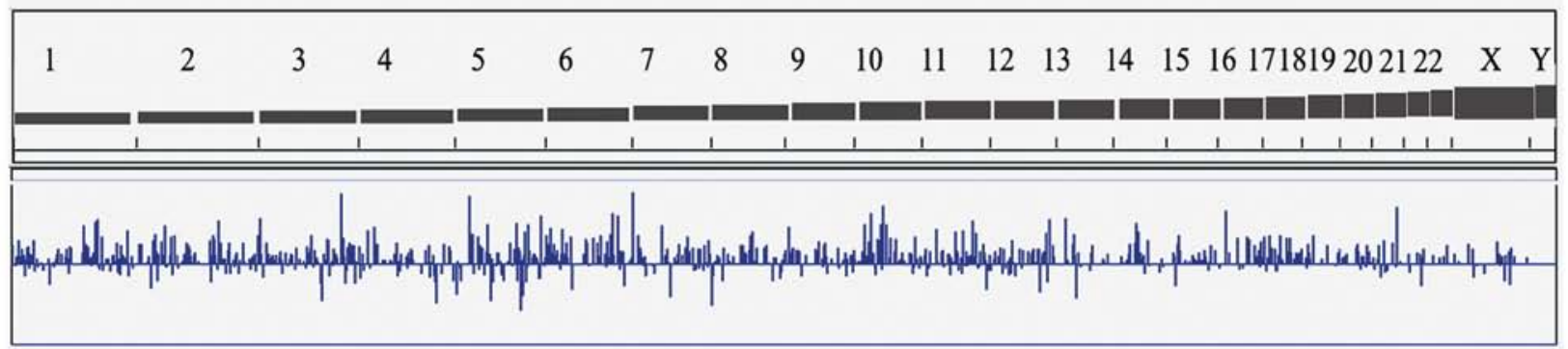

$\mathrm{C}$

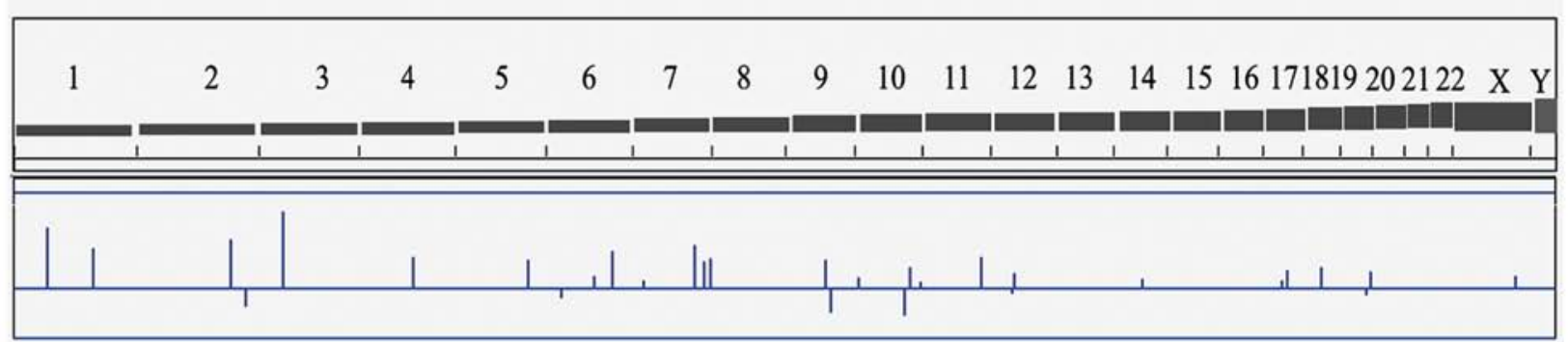

$\mathrm{D}$

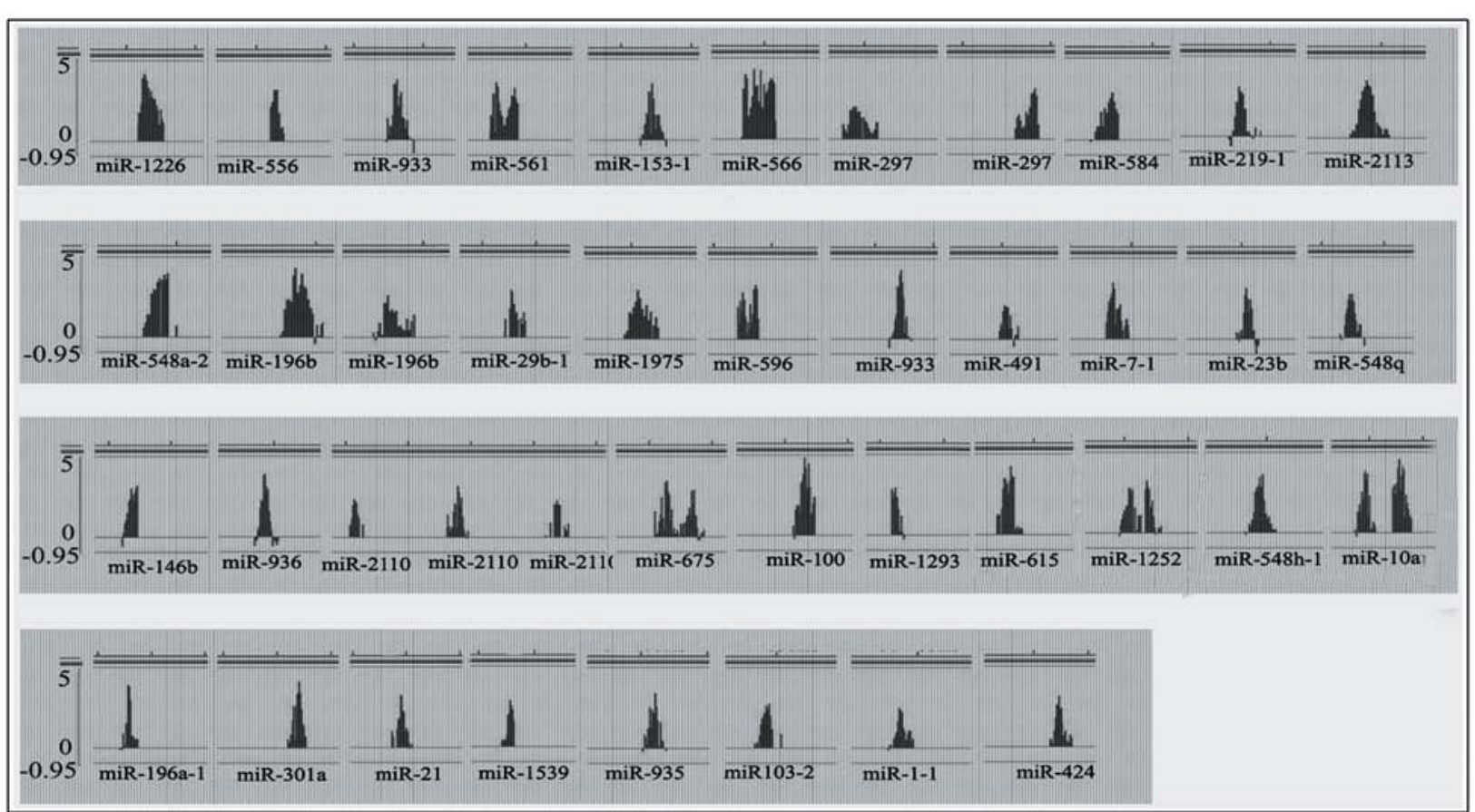

Figure 1. Distribution of TCF4 tiling array peaks within $10 \mathrm{~kb}$ of genes encoding protein or miRNA. High-confidence TCF4-binding sites (n=6,868), previously identified by Hatzis et al (1), were chosen for analysis. Binding site peaks located within $10 \mathrm{~kb}$ of transcription initiation sites were selected as likely to impact transcription regulation. (A) 1793 peaks distributed around coding genes, and 45 peaks distributed around miRNA. (B) Association of TCF4 binding sites with protein-coding genes was determined by TCF4 peak analysis. (C) Localization of TCF4 binding sites related to the nearest miRNA transcription region. (D) The distribution of TCF4 binding peaks corresponded to selected miRNA.

TCF4 activates miR-21 transcription by directly binding to its promoter. Tiling array analysis and ChIP-PCR identified miR-21 as a candidate TCF4 transcriptional target. MiR-21 expression is significantly enhanced in a variety of solid tumors, including 
A

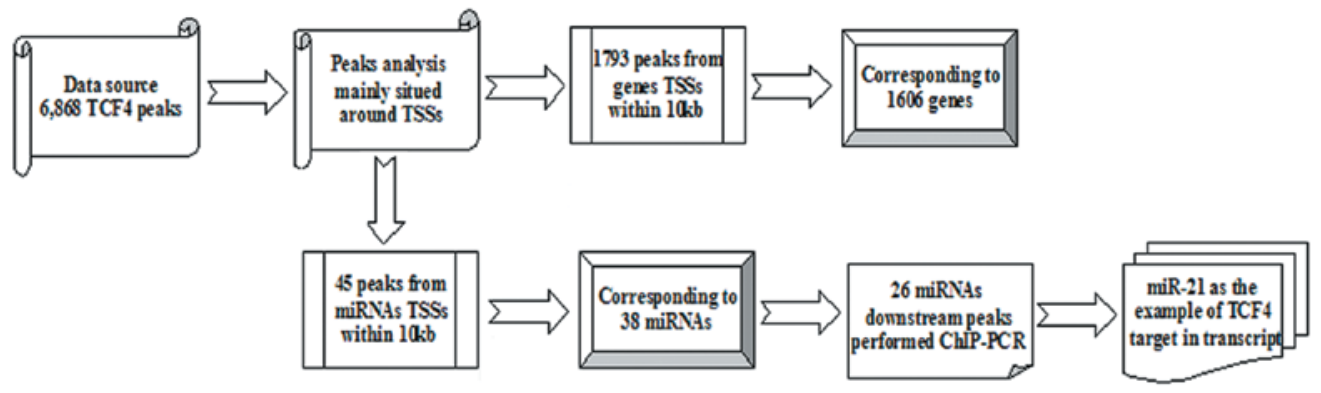

B

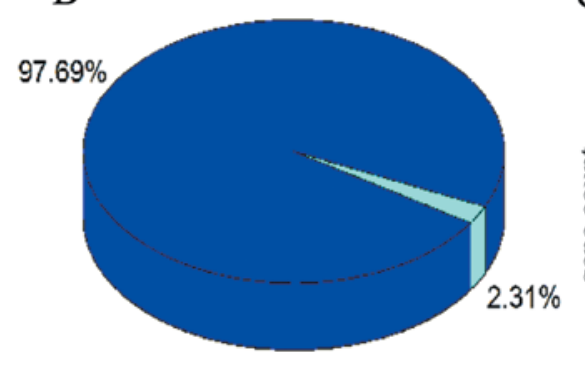

$\mathrm{C}$

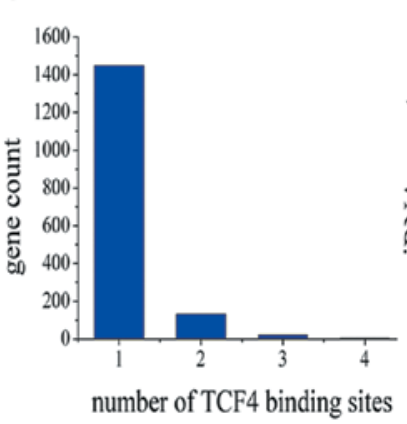

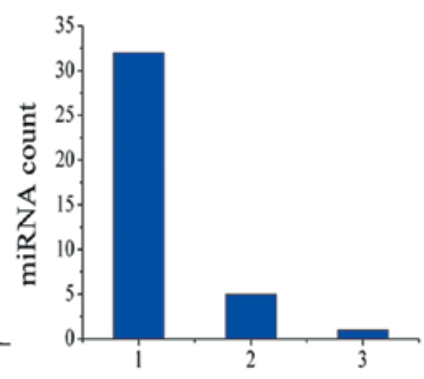

number of TCF4 binding sites

D
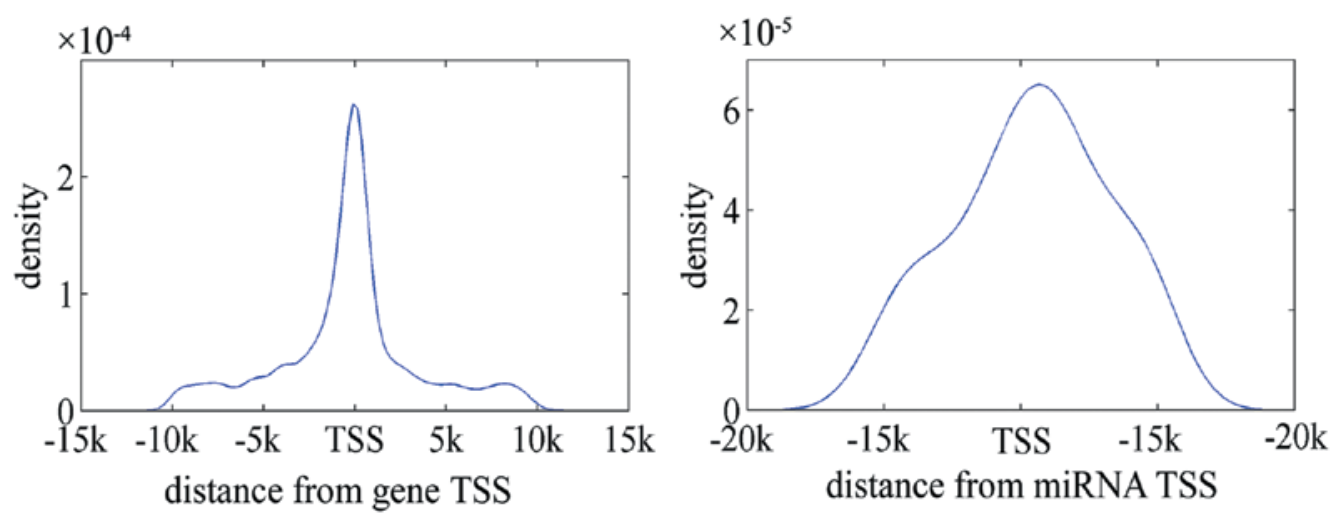

Figure 2. Tiling array analysis of TCF4 occupancy. (A) A graphical depiction of TCF4 regulates miRNA transcription via binding to their promoter regions. (B) The proportion of TCF4 peaks within $10 \mathrm{~kb}$ of protein-coding genes compared to miRNA is consistent with the ratio of known genes and miRNA. (C) TCF4 binding sites cluster around target genes. (D) TCF4 peaks situate around gene transcription initiation sites.

Table II. Nine miRNAs in tiling assays expressed in tumors compared with non-tumors.

\begin{tabular}{llll}
\hline miRNA & P-value & Fold change & $\begin{array}{c}\text { Fdr }(\%) \text { expression } \\
\text { state }\end{array}$ \\
\hline miR-196b & 0.74 & 1.05 & 37.03 \\
miR-196a-1 & 0.785 & 1.17 & 41.01 \\
miR-1-1 & 0.0005 & 0.942 & 0.364 \\
miR-100 & 0.319 & 1.15 & 19.25 \\
miR-21 & $<1 \times 10^{-7}$ & 1.7 & $<0.01$ \\
miR-103-2 & 0.845 & 1.28 & 45 \\
miR-29b-1 & 0.751 & 1.09 & 39 \\
miR-219-1 & 0.409 & 1.07 & 29.11 \\
miR-135-1 & NA & NA & NA \\
\hline
\end{tabular}

glioblastoma, breast, lung, colon, prostate, pancreas, and stomach cancers (16-21). Further, differential expression of miR-21 was observed in colon cancer, based upon tumor status, TNM staging, survival prognosis, and response to adjuvant chemotherapy (22). Although our results identify 9 miRNA regulated by TCF 4 , colon cancer tissue microarray containing 120 patient samples revealed that the only miRNA with differential expression in tumor vs. adjacent normal tissue was miR-21 (Table II). To determine whether TCF4 controls miR-21 transcription to regulate colon carcinogenesis, we detected the expression of miR-21 after blocking Wnt signaling pathway in LS174T cells. As anticipated, inhibition of Wnt signaling with aspirin suppressed miR-21 expression as determined by quantitative RT-PCR (qRT-PCR) and in situ hybridization. To specify the functional transcriptional regulation of miR-21 by Wnt/TCF4 


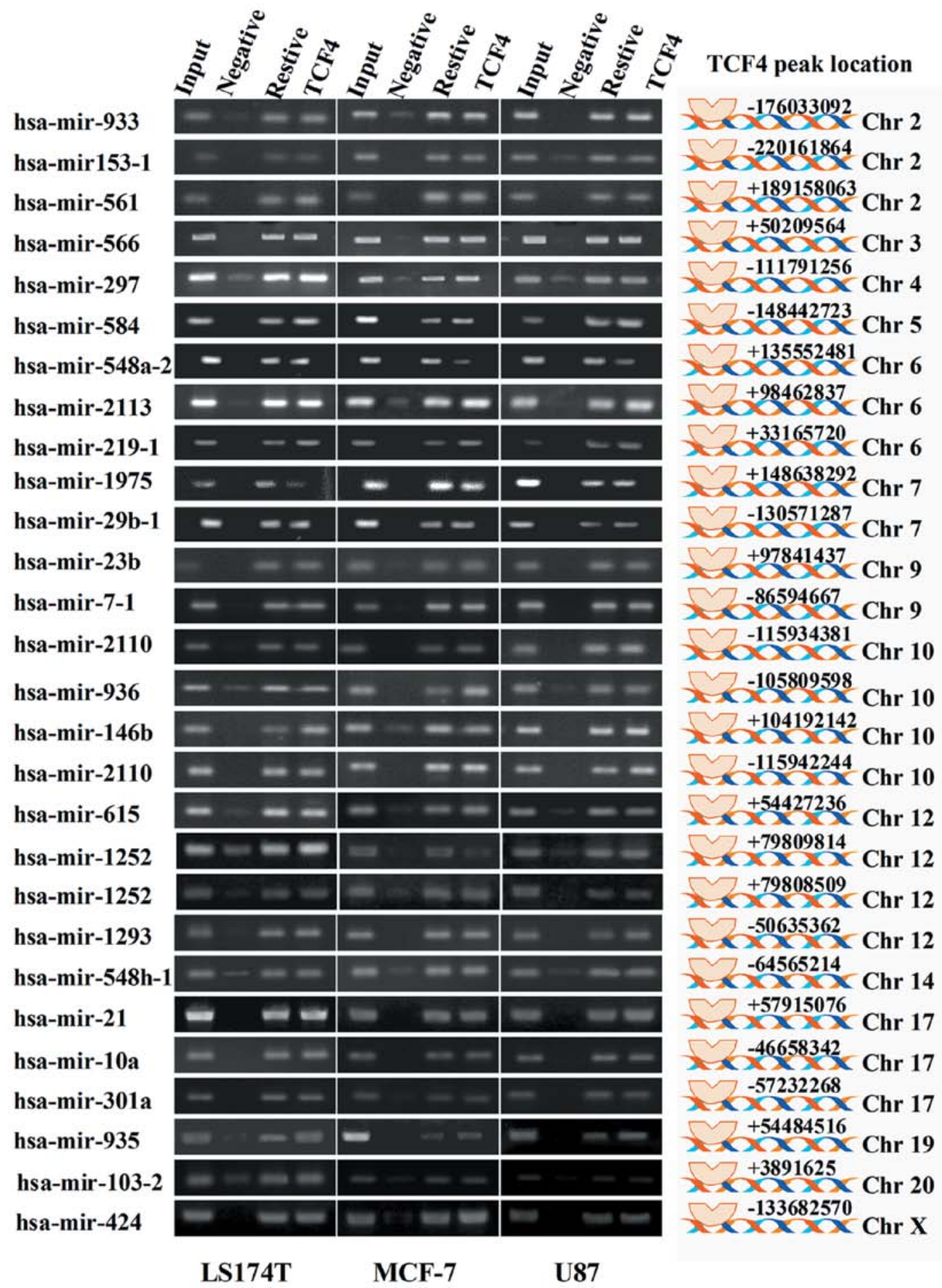

Figure 3. The distribution of 26 miRNA with transcription initiation sites located within $10 \mathrm{~kb}$ downstream of TCF4 binding sites. The association of TCF4 with the promoter regions of 26 miRNA was determined by ChIP PCR.

signaling, we designed a miR-21 luciferase reporter activated by a TCF4 binding motif upstream of its promoter region. Reporter assay revealed that TCF4 regulated miR-21 transcription by direct binding to the promoter. Western blotting was performed following Wnt inhibition, demonstrating a significant decrease in protein expression of miR-21 targets involved in cell proliferation, metastatic potential and tumorigenesis. In sum, these data identify Wnt/TCF4 regulation of miRNA-21 expression as a key driving force for aberrant protein expression during tumorigenesis and cancer progression.

\section{Discussion}

Wnt/ $\beta$-catenin/TCF signaling pathway has been shown to be a crucial factor in the development of many cancers. This complex regulates transcription of multiple genes involved in cellular proliferation, differentiation, survival and apoptosis, including Fra-1, c-myc and Cyclin D. In the past, our group has demonstrated that $\mathrm{Wnt} / \beta$-catenin/TCF signaling was significantly dysregulated in gliomas and down-regulated $\beta$-catenin/ TCF signaling which can inhibit cell proliferation and invasive 


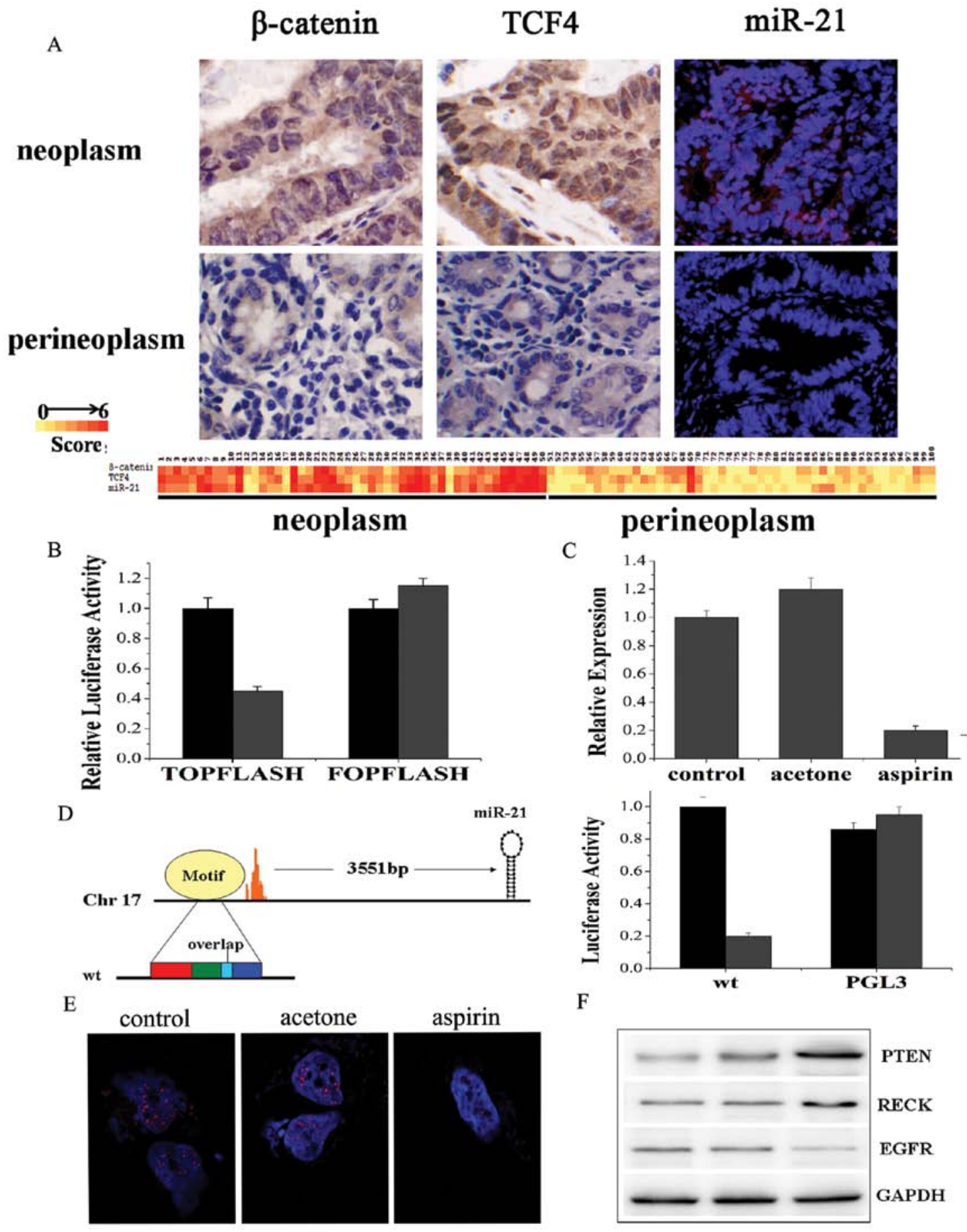

Figure 4. TCF4 regulates miR-21 expression. (A) TCF4 expression shows positive correlation with miR-21 expression, in a colon cancer tissue microarray derived from 100 patients. A heat map representing gene expression levels is shown, using a linear scale with a maximum immunoreactive score of 6 ( $<<0.01$ ). (B) Inhibition of $\beta$-catenin/TCF4-dependent transcription in response to aspirin treatment (10 mM, 4 h) was conducted by TOP-FOP experiment. (C) MiR-21 expression was suppressed in LS174T cells after $4 \mathrm{~h}$ treatment with $10 \mathrm{mM}$ aspirin compared to control and acetone treated cells, determined by qRT-PCR (D) The inhibition of $\beta$-catenin/TCF4 pathway by $10 \mathrm{mM}$ aspirin treatment resulted in decreased miR-21 transcription, as determined by luciferase reporter assay. (E) In situ examination detected miR-21 expression among no, actone, and $10 \mathrm{mM}$ aspirin treatment in LS174T colon carcinoma cells. (F) Cells treated with $10 \mathrm{mM}$ aspirin display changes in expression of miR-21 target proteins PTEN, RECK, and EGFR, as observed by Western blotting.

ability, induced apoptotic cell death (23). Furthermore, we clarified that down-regulation of $\beta$-catenin/TCF signaling inhibited several members of the EGFR pathway in human glioma cells (24). Association of deregulated Wnt/ $\beta$-catenin/TCF signaling for cancer treatment has thus generated significant interests. Accordingly, many documents suggest that $\beta$-catenin and TCF-4 play an important role in epithelial cancer progression and prognosis or prediction of response to therapy (25-27). Current drugs that target $\beta$-catenin/TCF complex have been reported such as Quercetin, FH535, and PKF118-310. However, in the present study we chose aspirin as the inhibitor the of the $\beta$-catenin/TCF complex, since many epidemiological studies have demonstrated aspirin can inhibit the transcriptional activity of Wnt targeted genes effectively in colon cancer cells, and the 
report which published in the Lancet elaborated daily aspirin reduced deaths of colon carcinoma during and after the trials supplied the patient evidence (28).

miRNA regulate gene expression by promoting or inhibiting the recruitment of RNA polymerase $(28,29)$, but the regulation of miRNAs in transcript was still unknown. The goal of the current study was to investigate whether the transcription factor TCF4 binds the promoter region of miRNA, regulating miRNA expression. Analysis of 6,868 TCF4 binding peaks previously identified using the genome wide ChIP-on-chip approach revealed that 1,793 biding sites were found in unique genes encoding protein, while 45 peaks were potentially found in the promoter region of miRNA. To demonstrate TCF4 interaction with a miRNA promoter, we employed ChIP-PCR to detect 26 miRNAs occupied downstream of TCF4 among U87, LS174T and MCF-7 cells. Mir-21 as one of the commonly implicated miRNAs in cancers is highly up-regulated and causally linked to proliferation, apoptosis, and migration of various cancer cell lines. Reporter assay confirmed a direct role for TCF4 in the regulation of miR-21 expression in LS174T colon carcinoma cells. Finally, we determined by tissue microarray that TCF4driven miR-21 expression increased with colon cancer stage.

In conclusion, this study demonstrated that the transcription factor TCF 4 can bind to the promoters of miRNAs and profoundly alter their expression. In past work, we demonstrated a role for miRNA in tumorigenesis, via binding to the seed sequence at the 3'-UTR (untranslated region) of target mRNAs and influencing the activity of the Wnt signaling pathway $(20,21,30,31)$. The present study for the first time provides evidence that the $\beta$-catenin/TCF signaling pathway can regulate numerous miRNAs transcription, and discovers the reason for miR-21 high expression in epithelial cancer. Collectively, these studies indicate that further investigation of the miRNA regulated by TCF4, and the potential impact of TCF4 gene silencing on both miRNA expression and tumorigenesis, are clearly warranted.

\section{Acknowledgements}

This work was supported by National Key Project of Science and Technology Supporting Programs of China (no. 2007BAI05B08), National Basic Research Project of China (2009CB918903), the China National Natural Scientific Fund (nos. 81001128, 30971136), the Program for New Century Excellent Talents in University (NCET-07-0615), the Tianjin Science and Technology Committee (09JCZDJC17600). The authors wish to express the gratitude to Shanghai Sensichip Co., Ltd., for bioinformatics analysis.

\section{References}

1. Hatzis P, van der Flier LG, van Driel MA, et al: Genome-wide pattern of TCF7L2/TCF4 chromatin occupancy in colorectal cancer cells. Mol Cell Biol 28: 2732-27344, 2008.

2. Miravet S, Piedra J, Miro F, Itarte E, Garcia de Herreros A and Dunach M: The transcriptional factor Tcf-4 contains different binding sites for beta-catenin and plakoglobin. J Biol Chem 277: 1884-18891, 2002.

3. Jin T and Liu L: The Wnt signaling pathway effector TCF7L2 and type 2 diabetes mellitus. Mol Endocrinol 22: 2383-2392, 2008.

4. Segditsas S and Tomlinson I: Colorectal cancer and genetic alterations in the Wnt pathway. Oncogene 25: 7531-7537, 2006.

5. Ozsolak F, Poling LL, Wang Z, et al: Chromatin structure analyses identify miRNA promoters. Genes Dev 22: 3172-3183, 2008.
6. Bartel DP: MicroRNAs: genomics, biogenesis, mechanism, and function. Cell 116: 281-297, 2004.

7. Vasudevan S, Tong Y and Steitz JA: Switching from repression to activation: microRNAs can up-regulate translation. Science 318: 1931-1934, 2007.

8. Esquela-Kerscher A and Slack FJ: Oncomirs - microRNAs with a role in cancer. Nat Rev Cancer 6: 259-269, 2006.

9. Cui Q, Yu Z, Purisima EO and Wang E: MicroRNA regulation and interspecific variation of gene expression. Trends Genet 23: 372-375, 2007.

10. Thomson JM, Newman M, Parker JS, Morin-Kensicki EM, Wright $\mathrm{T}$ and Hammond SM: Extensive post-transcriptional regulation of microRNAs and its implications for cancer. Genes Dev 20: 2202-2207, 2006.

11. Liu W, Zabirnyk O, Wang $\mathrm{H}$, et al: miR-23b targets proline oxidase, a novel tumor suppressor protein in renal cancer. Oncogene 29: 4914-4924, 2010

12. Wang KC, Garmire LX, Young A, et al: Role of microRNA-23b in flow-regulation of $\mathrm{Rb}$ phosphorylation and endothelial cell growth. Proc Natl Acad Sci USA 107: 3234-3239, 2010.

13. Fang Y, Shi C, Manduchi E, Civelek M and Davies PF: MicroRNA-10a regulation of proinflammatory phenotype in athero-susceptible endothelium in vivo and in vitro. Proc Natl Acad Sci USA 107: 13450-13455, 2010.

14. Huang H, Xie C, Sun X, Ritchie RP, Zhang J and Chen YE: miR-10a contributes to retinoid acid-induced smooth muscle cell differentiation. J Biol Chem 285: 9383-9389, 2010.

15. Fu X, Han Y, Wu Y, et al: Prognostic role of microRNA-21 in various carcinomas: a systematic review and meta-analysis. Eur $\mathrm{J}$ Clin Invest (In press).

16. Liu XG, Zhu WY, Huang YY, et al: High expression of serum miR-21 and tumor miR-200c associated with poor prognosis in patients with lung cancer. Med Oncol (In press).

17. Liu C, Li B, Cheng Y, et al: MiR-21 plays an important role in radiation induced carcinogenesis in $\mathrm{BALB} / \mathrm{c}$ mice by directly targeting the tumor suppressor gene Big-h3. Int J Biol Sci 7: 347-363, 2011.

18. Gong C, Yao Y, Wang Y, et al: Up-regulation of miR-21 mediates resistance to trastuzumab therapy for breast cancer. J Biol Chem 286: 19127-19137, 2011.

19. Tao J, Lu Q, Wu D, et al: microRNA-21 modulates cell proliferation and sensitivity to doxorubicin in bladder cancer cells. Oncol Rep 25: 1721-1729, 2011.

20. Zhou X, Ren Y, Moore L, et al: Downregulation of miR-21 inhibits EGFR pathway and suppresses the growth of human glioblastoma cells independent of PTEN status. Lab Invest 90: 144-155, 2010.

21. Zhou X, Zhang J, Jia Q, et al: Reduction of miR-21 induces glioma cell apoptosis via activating caspase 9 and 3. Oncol Rep 24: 195-201, 2010.

22. Schetter AJ, Leung SY, Sohn JJ, et al: MicroRNA expression profiles associated with prognosis and therapeutic outcome in colon adenocarcinoma. JAMA 299: 425-436, 2008.

23. Pu P, Zhang Z, Kang C, et al: Downregulation of Wnt 2 and betacatenin by siRNA suppresses malignant glioma cell growth. Cancer Gene Ther 16: 351-361, 2009.

24. Yue X, Lan F, Yang W, et al: Interruption of beta-catenin suppresses the EGFR pathway by blocking multiple oncogenic targets in human glioma cells. Brain Res 1366: 27-37, 2010.

25. MacDonald BT, Tamai K and He X: Wnt/beta-catenin signaling: components, mechanisms, and diseases. Dev Cell 17: 9-26, 2009.

26. Wei W, Chua MS, Grepper S and So SK: Blockade of Wnt-1 signaling leads to anti-tumor effects in hepatocellular carcinoma cells. Mol Cancer 8: 76, 2009.

27. Ying Y and Tao Q: Epigenetic disruption of the WNT/beta-catenin signaling pathway in human cancers. Epigenetics 4: 307-312, 2009.

28. Rothwell PM, Fowkes FG, Belch JF, et al: Effect of daily aspirin on long-term risk of death due to cancer: analysis of individual patient data from randomised trials. Lancet 377: 31-41, 2010.

29. Volinia S, Calin GA, Liu CG, et al: A microRNA expression signature of human solid tumors defines cancer gene targets. Proc Natl Acad Sci USA 103: 2257-2261, 2006.

30. Liu XS, Brutlag DL and Liu JS: An algorithm for finding protein-DNA binding sites with applications to chromatinimmunoprecipitation microarray experiments. Nat Biotechnol 20: 835-839, 2002.

31. Huang K, Zhang JX, Han L, et al: MicroRNA roles in betacatenin pathway. Mol Cancer 9: 252, 2010.

32. Zhang CZ, Zhang JX, Zhang AL, et al: MiR-221 and miR-222 target PUMA to induce cell survival in glioblastoma. Mol Cancer 9: 229, 2010. 